\title{
Editorial
}

\section{Geht Kirche digital?}

https://doi.org/10.1515/mdki-2021-0033

Liebe Leserinnen und Leser,

die Corona-Pandemie hat der allgemeinen Digitalisierung der Welt - und damit auch der Kirchen - einen starken Schub verpasst. Und dementsprechend findet sich das Thema verstärkt in der einschlägigen Literatur. Aus unzähligen Perspektiven wurde diese Frage auch schon vor Corona diskutiert, wurden die positiven und negativen Seiten angesprochen. ${ }^{1}$ Aus dem kirchlichen Bereich seien hier aus jüngster Zeit nur zwei Veröffentlichungen erwähnt: die EKD-Publikation Freiheit digital. Die zehn Gebote in Zeiten des digitalen Wandels. Eine Denkschrift der Evangelischen Kirche in Deutschland (2021) sowie die erweiterte zweite Auflage von Werner Thiedes Buch Digitaler Turmbau zu Babel. Der Technikwahn und seine Folgen (2021). Beide nehmen die ethischen Fragen auf, die sich mit den neuen Techniken stellen, bzw. beschäftigen sich mit den Risiken, die mit den damit verbundenen Änderungen auf die Menschheit zukommen.

So mag es scheinen, dass der MdKI mit diesem Heft nicht viel Neues bieten kann. Dennoch haben wir uns entschlossen, dieses Thema aufzugreifen und aus konfessionskundlicher Perspektive die theologische bzw. ekklesiologische Frage zu stellen: Geht Kirche digital? Ist eine Kirche, in der sich die Mitglieder „nur“ digital treffen, tatsächlich Kirche? Aus den praktischen Erfahrungen, die die verschiedenen Kirchen in der Pandemie gemacht haben, könnte man sehr unterschiedliche Antworten darauf erwarten. Vielleicht für manche eine Überraschung: Die Antworten, die in diesem Heft aus verschiedenen konfessionellen Blickwinkeln gegeben werden, können praktisch unisono zusammengefasst werden mit „Ja, aber ...“ Am deutlichsten in Richtung „Ja, Kirche geht digital“ ist der römisch-katholische Beitrag von Andreas Büsch. Überraschen mag auch der orthodoxe Beitrag von Alexander

1 Instruktiv dafür ist der umfangreiche Literaturüberblick, den Werner Thiede, Digitalisierungsrisiken und Fortschrittsglaube. Literaturumschau innerhalb wie außerhalb von Theologie und Kirche, in ThR 84 (2019), 260-316, bietet.
Ponomariov, der die Möglichkeit einer hybriden Eucharistie aufzeigt. Dagegen ist - vermutlich auch entgegen bestimmten Erwartungen - die freikirchliche Stimme von Arndt Schnepper durchaus ambivalent in ihrer Antwort. Aus der Erfahrung des multilateralen und internationalen ökumenischen Betens, wie es im Ökumenischen Zentrum in Genf (dem Sitz des Ökumenischen Rates der Kirchen und anderer ökumenischer Organisationen) täglich praktiziert wird, berichtet und reflektiert Mikie Roberts und findet die „Ekklesia“ auch im digitalen Format. Die Zwiespältigkeit von positiven neuen Möglichkeiten digitaler Formate bei gleichzeitig fehlender Gemeinschaft bestätigt auch der Rückblick von Verena Hammes auf den zum großen Teil digital durchgeführten 3. Ökumenischen Kirchentag.

Aus aktuellem Anlass geben wir in diesem Heft auch dem neuen Präsidenten des Evangelischen Bundes, Dr. h.c. Christian Schad, Gelegenheit, seine Überlegungen zu jüngsten Texten aus dem evangelisch-katholischen Gespräch mit unseren Leserinnen und Lesern zu teilen. Sie sind gedacht als Impuls für die Fortführung der Diskussionen um das gemeinsame Abendmahl und die damit verbundene Frage der gegenseitigen Anerkennung der Ämter.

Drei Rezensionen nehmen weitere ökumenische Themen auf und stellen neueste Literatur dazu vor: Christine Lienemann-Perrin bespricht die Darstellung der Überlegungen zum Konzept des Globalen Christentums bzw. World Christianity. Dagmar Heller präsentiert eine Veröffentlichung über Faith and Science in Russian Religious Thought, die aufschlussreich für das Nachdenken über Glauben und Vernunft in der russischen Orthodoxie ist, und Dirk Spornhauer bespricht eine Publikation des Bundes Freikirchlicher Pfingstgemeinden, in der neue Entwicklungen im Hinblick auf Fragen der Leitung in der Pfingstbewegung deutlich werden.

Wir wünschen - wie immer auch im Namen unserer Redaktionskolleg*innen Martin Bräuer und Miriam Haar eine gute Lektüre!

Dagmar Heller und Lothar Triebel 